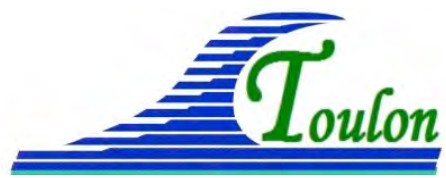

XIV èmes Journées Nationales Génie Côtier - Génie Civil Toulon, 29 juin au $1^{\text {er }}$ juillet 2016

DOI:10.5150/jngcgc.2016.047 @ Editions Paralia CFL

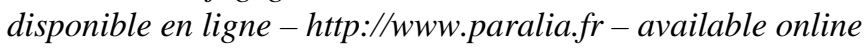

\title{
Comparaison de formules pour le calcul des forces mécaniques dues à la houle sur des bâtiments émergés
}

\author{
Florian BREHIN ${ }^{1}$, Nicolas ZIMMERMANN ${ }^{1}$, \\ Vincent GRUWEZ ${ }^{1}$, Annelies BOLLE ${ }^{1}$
}

\author{
1. International Marine \& Dredging Consultants (IMDC) NV, \\ Van Immerseelstraat 66, 2018 Anvers, Belgique. \\ florian.brehin@imdc.be
}

\section{Résumé :}

Cet article présente l'application de plusieurs formules pour le calcul des forces mécaniques dues à l'action de la houle sur des bâtiments émergés. Il illustre le run-up dû à la houle, les forces horizontales (Fh) ainsi que les limites de la méthodologie de calcul avec une application sur plusieurs profils du littoral Français. Le choix des formules est basé sur des recherches bibliographiques et inclut: Goda-Takahashi, Camfield, Pedersen (USACE, 2006), Chen (CHEN, 2012 ; CHEN et al., 2015), et une formule théorique fondée sur le guide Eurotop (PULLEN et al., 2007), développée pour cette étude. Chaque formule est caractérisée par un domaine d'application particulier, un comportement asymptotique, et des données d'entrée spécifiques qui sont discutées en détail dans cet article.

Les résultats confirment qu'il n'existe pas de formule universellement valable pour l'ensemble des configurations du littoral. Différentes formules dans leurs domaines d'applicabilité diffèrent typiquement de $\pm 30 \%$ ou plus. La formule de CHEN et al. (2015) a le domaine d'application le plus important pour des bâtiments émergés. La formule de Camfield est utile pour les plages de faible pente. De manière surprenante, la formule théorique fondée sur le guide Eurotop prédit des résultats raisonnables sur un grand spectre de configurations. Pour obtenir un premier ordre de grandeur des forces exercées par la houle sur un bâtiment émergé, il est donc recommandé d'estimer une enveloppe moyenne ou maximale des formules applicables et d'utiliser la formule théorique fondée sur le guide Eurotop.

Mots-clés : Forces mécaniques, Run-up, Débits franchissement, Risques d’inondation, Vendée.

\section{Introduction}

Dans le cadre d'une étude de risques littoraux dans le Nord-Ouest de la France, il a été demandé d'estimer les forces mécaniques exercées par la houle sur des bâtiments émergés situés sur le littoral. Le but de cet article est de présenter des outils simples et rapides d'utilisation pour des ingénieurs travaillant dans des bureaux d'étude. Il s'agit 


\section{Thème 4 - Ouvrages portuaires, offshore et de plaisance}

essentiellement de formules semi-empiriques voire empiriques qui sont présentées et comparées.

La littérature distingue quatre types d'impact de houle: (i) non déferlante ; (ii) en début de déferlement à impact maximal ("impact load") ; (iii) déferlante avec emprisonnement d'une poche d'air ; (iv) et déferlée (run-up) au-dessus du niveau marin. Un aperçu des formules de calcul des forces de houle sur des structures est présenté par KORTENHAUS et al. (1998) dans le cadre du projet PROVERBS. Cet aperçu, très complet pour l'époque, reste d'actualité et contient les principales formules reprises dans le Coastal Engineering Manual (USACE, 2006 ; chapitre 5) et le Rock Manual (CIRIA et al., 2009 ; paragraphe 5.2.2.12).

Les formules de calcul de pression et de force dues à la houle ont historiquement été développées pour la conception de brise-lames en caissons. Il s’agit essentiellement de la pression exercée sur une structure immergée par une houle déferlante en pied de structure, par profondeur en général importante.

Plusieurs formules ont également été développées pour la stabilité des murs de couronnement de ces mêmes brise-lames, typiquement situés au-dessus de la ligne d'eau. Cette situation est déjà plus proche du cas étudié, à la différence majeure que le mur de couronnement n'absorbe pas la totalité du choc de la houle car il est de faible hauteur et des débits franchissant résiduels sont présents. Ces formules sous-estiment donc la pression par rapport à un mur infini dans la même configuration. De même les bâtiments se situent en général beaucoup plus à l'intérieur des terres qu'un mur de couronnement. Enfin le brise-lames n'est pas une situation représentative du profil de plage que l'on trouve en général devant les bâtiments.

Les formules présentant le plus d'intérêt pour cette étude correspondent aux derniers développements en la matière, notamment les travaux de l'université de Gand et de l’institut Flanders Hydraulics, sur l'impact des houles déferlées sur des bâtiments situés sur une digue.

Compte tenu du grand nombre de configurations réelles à étudier, plusieurs formules ont été appliquées. Le choix des formules repose sur une étude bibliographique. Une traduction est aussi proposée des vitesses et profondeurs des débits franchissant du guide Eurotop (PULLEN et al., 2007) en une force. Bien que cette approche soit souhaitée par une grande partie de la communauté scientifique, la réalité est bien plus complexe. Chaque formule est caractérisée par un domaine d'application particulier, un comportement asymptotique hors de leur domaine d'application, et des données d'entrée géométriques et hydrodynamiques très spécifiques. Ces différences sont illustrées, et des recommandations sont faites en conclusion sur l'approche à suivre pour une telle application. Les bâtiments sont schématisés par un mur infiniment haut. 


\section{XIV èmes Journées Nationales Génie Côtier - Génie Civil \\ Toulon, 29 juin au $1^{\text {er }}$ juillet 2016}

\section{Méthodologie}

\subsection{Zone d'étude}

La Vendée est située à la limite sud du bassin armoricain et est caractérisée par une géologie et une géomorphologie côtières variables avec de nombreux ouvrages de protection côtière (enrochements, épis, murs de soutènement, etc.) dont la géométrie doit être prise en compte pour les calculs de forces. Les conditions de houle sont assez énergétiques, notamment durant les tempêtes hivernales de l'Atlantique Nord et peuvent être accompagnées de surcotes. Les niveaux d'eau maximum peuvent atteindre les $+4 \mathrm{~m}$ IGN69 pour des périodes de retour centennales (ALP’GEORISQUES \& IMDC, 2014).

\subsection{Sélection des formules}

Les formules suivantes ont été sélectionnées (une par cas):

- Goda (1985, in USACE, 2006) amendée par TAKAHASHI et al. (1994) pour le calcul des forces sur un caisson immergé (houle quasi-statique ou impactante), car c'est la formule la plus populaire pour ce type d'application ;

- Camfield (1991, in USACE, 2006) pour la force due au run-up sur un bâtiment situé sur une plage à faible pente, car il s'agit de la seule formule existante pour cette application ;

- Pedersen (1997, in USACE, 2006) pour le calcul des forces sur un mur de couronnement (houle déferlée), car c'est la formule la plus fiable pour ce type d'application selon le Rock Manual (CIRIA et al., 2009) ;

- CHEN (2012) et CHEN et al. (2015) pour le calcul des forces dues au run-up sur un bâtiment situé sur la crête d'une digue, car ce sont les seules formules pour ce type d'application ;

- Eurotop (PULLEN et al., 2007) qui ne permet pas de calculer directement la force mais qui permet une estimation de la vitesse $\left(\mathrm{v}_{\mathrm{A}}\right)$ et de la profondeur $\left(\mathrm{h}_{\mathrm{A}}\right)$ de run-up sur la pente côté mer et sur la crête de l'ouvrage. Ces paramètres sont traduits en une force horizontale théorique $\mathrm{F}_{\mathrm{h}}$ par mètre linéaire $[\mathrm{N} / \mathrm{m}]$ :

$F_{h}=\rho v_{A}^{2} h_{A}$

Chaque formule utilise des paramètres hydrodynamiques et des formules de run-up différentes. Comme ces formules ont été calibrées sur leur série spécifique de tests, il est important de ne pas les harmoniser (par exemple en considérant la même formule de run-up). Le tableau 1 résume les paramètres d'entrée des différentes formules.

Le tableau 2 résume les domaines d'applicabilité retenus pour l'étude par rapport aux paramètres effectivement testés lors de l'élaboration de ces formules. 


\section{Thème 4 - Ouvrages portuaires, offshore et de plaisance}

Tableau 1. Paramètres d'entrée pour les différentes formules. Hs $=$ hauteur significative de houle, Hmax = hauteur maximale de houle, $k s i=$ paramètre de surf.

\begin{tabular}{|c|c|c|c|c|c|}
\hline Formule & Base & Pente & Run-up & Franc-bord & Distance \\
\hline Goda & $\begin{array}{l}\text { tests à } \\
\text { échelle } \\
\text { réduite }\end{array}$ & $\begin{array}{l}\text { brise- } \\
\text { lames }\end{array}$ & hauteur maximale d'impact & $\begin{array}{l}\text { profondeur en } \\
\text { pied de caisson } \\
(<0 \mathrm{~m})\end{array}$ & $\begin{array}{l}\text { crête du brise- } \\
\text { lames, berme } \\
\text { sous l'eau }\end{array}$ \\
\hline Pedersen & $\begin{array}{l}\text { tests à } \\
\text { échelle } \\
\text { réduite }\end{array}$ & $\begin{array}{l}\text { brise- } \\
\text { lames }\end{array}$ & $\begin{array}{l}0,1 \% \text { de dépassement } \\
1,34 \times H s \times k s i^{0,55}\end{array}$ & $\begin{array}{l}\text { crête du brise- } \\
\text { lames }\end{array}$ & $\begin{array}{l}\text { crête du brise- } \\
\text { lames }\end{array}$ \\
\hline Camfield & théorique & plage & $\begin{array}{l}\text { run-up max. de Schüttrümpf } \\
2,25 \times \operatorname{Hmax} \times \tanh (0,5 \times k s i)\end{array}$ & $\begin{array}{l}\text { pas de franc- } \\
\text { bord, pente } \\
\text { continue }\end{array}$ & trait de côte \\
\hline $\begin{array}{l}\text { CHEN } \\
(2012)\end{array}$ & $\begin{array}{l}\text { tests à } \\
\text { échelle } \\
\text { réduite }\end{array}$ & digue & $\begin{array}{l}\text { run-up max. de Schüttrümpf } \\
2,25 \times \text { Hmax } \times \tanh (0,5 \times k s i)\end{array}$ & crête de digue & crête de digue \\
\hline $\begin{array}{l}\text { CHEN et } \\
\text { al. (2015) }\end{array}$ & $\begin{array}{l}\text { tests à } \\
\text { échelle } \\
\text { réduite }\end{array}$ & digue & $\begin{array}{l}\text { run-up max. de Schüttrümpf } \\
2,25 \times \text { Hmax } \times \tanh (0,5 \times k s i)\end{array}$ & crête de digue & crête de digue \\
\hline Eurotop & théorique & digue & $\begin{array}{l}2 \% \text { de dépassement; } \\
1,65 \times H s \times k s i\end{array}$ & crête de digue & trait de côte \\
\hline
\end{tabular}

Tableau 2. Domaines d'applicabilité retenus pour l'étude par rapport aux paramètres effectivement testés lors de l'élaboration de ces formules.

\begin{tabular}{|c|c|c|c|}
\hline Formule & Pente $(\tan \alpha)$ & Franc-bord (m) & Largeur de crête (m) \\
\hline Goda & $\begin{array}{l}\text { 1:0,5 à 1:4 } \\
\text { (testé inconnu) }\end{array}$ & $\begin{array}{l}-5 m \text { à }-0,5 m \\
\text { (testé }-3,9 m \text { à }-0,7 m \text { ) }\end{array}$ & $\begin{array}{l}0 \text { m à } 25 \mathrm{~m} \\
\text { (testé } 0,25 \mathrm{~m} \text { à } 20 \mathrm{~m} \text { ) }\end{array}$ \\
\hline Pedersen & $\begin{array}{l}1: 1 \text { to } 1: 3,7 \\
\text { (testé } 1: 1,5 \text { à } 1: 3,5 \text { ) }\end{array}$ & $\begin{array}{l}1,5 \mathrm{~m} \text { to } 5,0 \mathrm{~m} \\
\text { (testé } 2,2 \mathrm{~m} \text { à } 3,8 \mathrm{~m} \text { ) }\end{array}$ & $\begin{array}{l}2,5 m \text { à 7,5 m } \\
\text { (testé 3,6 m à 7,2 m) }\end{array}$ \\
\hline Camfield & $\begin{array}{l}\text { 1:10 à } 1: 100 \\
\text { (théorique } 1: 10 \text { à } 1: 100 \text { ) }\end{array}$ & NA & $N A$ \\
\hline CHEN (2012) & $\begin{array}{l}1: 2 \text { à } 1: 3 \\
\text { (testé } 1: 2,35)\end{array}$ & $\begin{array}{l}\text { 1,5 m à 3,0 m } \\
\text { (testé inconnu) }\end{array}$ & $\begin{array}{l}0 \mathrm{~m} \text { à } 15 \mathrm{~m} \\
(\text { testé } 0 \mathrm{~m} \text { et } 15 \mathrm{~m})\end{array}$ \\
\hline CHEN et al. (2015) & $\begin{array}{l}\text { 1:1,5 à } 1: 8 \\
\text { (testé } 1: 3 \text { à 1:6) }\end{array}$ & $\begin{array}{l}0 m \text { à 2,5 m } \\
\text { (testé 0,4 m à 1,5 m) }\end{array}$ & $\begin{array}{l}5 m \text { à } 25 \mathrm{~m} \\
\text { (testé } 7,5 \mathrm{~m} \text { à 22,5 m) }\end{array}$ \\
\hline Eurotop & $\begin{array}{l}1: 1 \text { à } 1: 10 \\
\text { (testé } 1: 1 \text { à } 1: 8 \text { ) }\end{array}$ & $\begin{array}{l}0 \mathrm{~m} \text { à 5,0 m } \\
\text { (testé inconnu) }\end{array}$ & $N A$ \\
\hline
\end{tabular}

Le comportement asymptotique a aussi été étudié pour anticiper les résultats hors de leur domaine d'applicabilité. La formule de Goda est indépendante de la pente et prédit une force constante non nulle à une distance infinie, avec un comportement non réaliste pour un franc-bord positif. La formule n'est jamais applicable dans le cas présent. La formule de Pedersen est non définie si la pente est inférieure à 1:3,7. La force tend vers l'infini pour une distance de 0 . La formule de Camfield considère une pente infiniment 


\section{XIV ${ }^{\text {èmes }}$ Journées Nationales Génie Côtier - Génie Civil \\ Toulon, 29 juin au $1^{\text {er }}$ juillet 2016}

longue. Cette hypothèse est remplacée par une hypothèse de force constante après la crête. La formule théorique d'Eurotop est la seule à avoir un comportement asymptotique raisonnable pour chacun des trois paramètres (pente, distance et francbord). La formule de CHEN et al. (2015) est caractérisée par une force qui tend vers moins l'infini si la distance est de 0 . Des limiteurs ont été ajoutés pour garantir des résultats réalistes hors de leur domaine d'applicabilité.

\subsection{Paramètres d'entrée}

Les données de profils de plage correspondent aux données d'entrée pour les calculs de franchissement de l'étude PPRL (ALP'GEORISQUES \& IMDC, 2014). Les données hydrodynamiques sont issues de simulations numériques des modèles SWAN et Delft3D, pour la houle et les niveaux d'eau, respectivement (ALP'GEORISQUES \& IMDC, 2014). Le scenario modélisé correspond à la tempête Xynthia (février 2010) avec changement climatique.

\section{Résultats}

Les résultats de calcul des forces pour les conditions hydrodynamiques correspondant au scénario Xynthia +0,20 m sont présentés pour un profil représentatif (figure 1). Les forces calculées sont présentées sous forme de graphiques de la force en fonction de la distance entre le début la crête et le bâtiment, représentant à la fois les courbes de forces individuelles mais également la force "enveloppe" de couleur rose correspondant à la moyenne des formules applicables là où il y en a au moins une. Les forces individuelles sont marquées d'un trait fin lorsqu'elles ne sont pas applicables et d'un trait épais lorsqu'elles le sont. Si aucune formule n'est applicable l'enveloppe n'est pas définie. C’est le cas de plusieurs profils caractérisés par des valeurs de run-up trop importantes calculées à cause d'une pente trop forte. La légende inclut les valeurs de run-up, ainsi que les valeurs du paramètre de surf (ksi) calculées pour chacune des formules.

\section{Discussion}

Les résultats illustrent la grande incertitude autour des forces calculées par différentes formules, même lorsqu'elles sont dans leur domaine d'applicabilité. Cette incertitude est typiquement de $\pm 30 \%$ autour de la valeur moyenne, parfois plus. Ils illustrent aussi l'impossibilité d'utiliser une unique formule couvrant l'ensemble des configurations réelles. Pour la zone étudiée, la formule de CHEN et al. (2015) est la plus souvent applicable. De manière surprenante, la formule simpliste fondée sur le guide Eurotop donne un ordre de grandeur très réaliste pour un grand spectre de configurations réelles. Les calculs de run-up pour Camfield sont surestimés pour la plupart des profils du fait du manque d'applicabilité à cause d'une pente trop forte. La formule de Goda, bien que jamais applicable, coïncide de manière surprenante avec les formules applicables dans les cas avec une forte pente et un franc-bord faible, au moins sur la première vingtaine 
de mètres à partir de la crête. Les formules de CHEN (2015) et Eurotop, bien que leurs domaines d'applicabilité ne couvrent qu'une partie du profil, semblent donner les meilleurs résultats d'estimations de forces, avec une bonne correspondance dans les calculs des forces pour ces deux formules. Les résultats paraissent particulièrement réalistes lorsque les forces se recoupent.
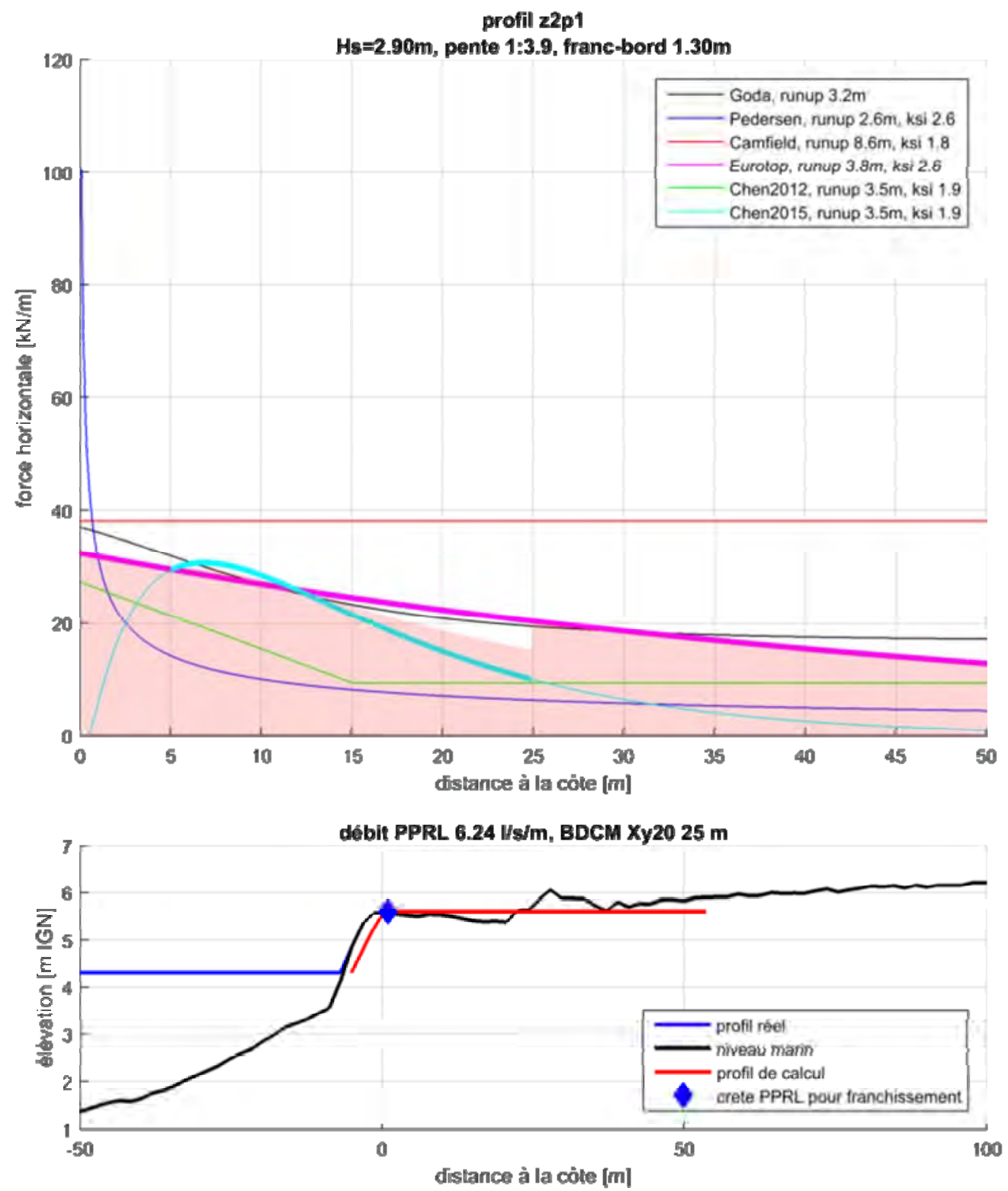

Figure 1. Exemple de résultats de calcul des forces pour un profil : forces individuelles (traits) et force "enveloppe" (couleur rose).

Pour les profils plus pentus (1:1 à 1:10), la formule de Pedersen donne des résultats satisfaisants, car la force en haut du mur de couronnement (même si on est en dehors de l'applicabilité) recoupe les valeurs des forces de Eurotop et CHEN et al. (2015). A noter 


\section{XIV èmes Journées Nationales Génie Côtier - Génie Civil \\ Toulon, 29 juin au $1^{\text {er }}$ juillet 2016}

que CHEN et al. (2015) n'est en réalité valable que sur une distance d'environ $20 \mathrm{~m}$ à partir de la crête. A l'inverse, Camfield ne s'applique que pour une pente faible (1:10 à 1:100). L'incertitude dans cette formule est importante car il s'agit d'une formule théorique qui n'a pas été calibrée sur des mesures.

Globalement, les formules de CHEN et al. (2015) et Eurotop donnent des estimations raisonnables pour des profils caractérisés par des pentes comprises entre 1:1 et 1:10 et des franc-bords entre $1 \mathrm{~m}$ et $3 \mathrm{~m}$. La formule Eurotop est particulièrement flexible et offre un domaine de validité qui recoupe la majeure partie du profil. Néanmoins le domaine de validité d'Eurotop est lié aux formules pour la vitesse et la profondeur de run-up (hauteur d'eau depuis la crête du profil) seulement. Même si ces deux valeurs sont correctes, le fondement théorique de cette formule de conversion en une force est discutable, car elle n’a pas encore été validée par la littérature scientifique. Pour les pentes plus faibles que 1:10, la formule de Camfield peut être utilisée pour estimer la force en début de crête.

Les formules ci-dessus permettent la détermination d'ordres de grandeur seulement. Afin d'aboutir à une quantification précise des forces mécaniques, il est nécessaire de procéder à des essais en bassin (modélisation physique) ou bien à des modélisations numériques complexes (Dynamique des Fluides Computationnelle ou CFD).

\section{Conclusions}

Cette étude a permis le calcul des forces horizontales exercées par la houle sur un mur infiniment haut, pour une série de configurations très différentes du littoral vendéen.

Les résultats confirment qu'il n'existe pas de formule universellement valable pour l'ensemble des configurations du littoral. Différentes formules dans leurs domaines d'applicabilité diffèrent typiquement de $\pm 30 \%$ ou plus.

Pour obtenir un premier ordre de grandeur des forces exercées par la houle sur un bâtiment émergé, il est donc recommandé d'estimer une enveloppe moyenne ou maximale des formules applicables et d'utiliser la formule théorique fondée sur le guide Eurotop.

Enfin, la traduction réglementaire de calculs de forces dues à la houle se heurte à deux obstacles. La première est l'incertitude des résultats liée à un manque de connaissances scientifiques des forces dues à la houle sur un bâtiment émergé. La deuxième est la nécessité de définir des limites de résistances adéquates, en fonction des divers paramètres définissant l'ouvrage (exposition, matériau, caractéristiques structurelles, modes de défaillance, ....). Cette traduction, qui n'a pas fait l'objet de cette étude, est loin d'être évidente. En effet, un bâtiment est conçu pour une force déterminée, tandis qu'ici on fait l'inverse: étant donné une structure existante, le problème étant de déterminer la force majorante pour laquelle il a été conçu. 
Thème 4-Ouvrages portuaires, offshore et de plaisance

\section{Références bibliographiques}

ALP'GÉORISQUES \& IMDC (2014). Plan de Prévention des Risques Littoraux en Vendée. DDTM 85. Rapport de Phase 1.

CHEN X., HOFLAND B., ALTOMARE C., SUZUKI T. (2015). Forces on a vertical wall on a dike crest due to overtopping flow. Coastal Engineering, Vol. 95, pp 94-104. http://dx.doi.org/10.1016/j.coastaleng.2014.10.002

CHEN X. (2012). Hydrodynamic loads on buildings caused by overtopping waves. Master Thesis for Delft University of Technology, Delft, Netherlands; Project number: 770_59 for Flanders Hydraulics Research, Antwerp, Belgium.

CIRIA, CUR \& CETMEF (2009). The Rock Manual. The use of rock in hydraulic engineering (2nd edition). C683, CIRIA, London.

KORTENHAUS A., OUMERACI H., ALLSOP N.W.H., WALKDEN M., MULLER G., CALABRESE M., VICINANZA D. (1998). MAST III/PROVERBS. Chapter 5.1. Wave impact loads - Pressures and Forces - PROVERBS (Probabilistic Design Tools for Vertical Breakwaters), contract MAS3-CT-0041. Final Report

PULLEN T., ALLSOP N.W.H., BRUCE T., KORTENHAUS A., SCHÜTTRUMPF H., VAN DER MEER J.W. (2007). Wave overtopping of sea defences and related structures: assessment manual. EurOtop.

TAKAHASHI S., TANIMOTO K, SHIMOSAKO K. (1994). A proposal of impulsive pressure coefficient for design of composite breakwaters. Proc. of Int. Conf. on HydroTechnical Eng. For Port and Harbor Construction. Yokosuka, Japan.

USACE -U.S. Army Corps of Engineers- (2006). Coastal Engineering Manual. Manual 1110-2-1100, U.S.A.C.E, Washington, D.C. (in 6 volumes). 